\title{
A NOVEL NON-LINEAR DISCRETE HOMOGENIZATION APPROACH FOR THE ANALYSIS OF DOUBLE CURVATURE MASONRY STRUCTURES
}

\author{
J. SCACCO ${ }^{1 *}$, G. MILANI ${ }^{1}$ AND P.B. LOURENÇO ${ }^{2}$ \\ ${ }^{1}$ Politecnico di Milano, Department of Architecture Built Environment and Construction \\ Engineering ABC, Piazza Leonardo da Vinci 32, 20133 Milan, Italy \\ e-mail: jacopo.scacco@polimi.it (*corresponding author) \\ gabriele.milani@polimi.it \\ 2 Department of Civil Engineering, ISISE, University of Minho, \\ Azurém, 4800-058 Guimarães, Portugal \\ email: pbl@civil.uminho.pt
}

Keywords: Masonry, Dome, Homogenization, Automatic Mesh, Non-linear Analysis

\begin{abstract}
In the context of historical heritage, curved masonry structures as arches, vaults and domes represent the most distinctive and charming feature. Since the $17^{\text {th }}$ century, several approaches have been developed in order to analyze their behavior, achieving nowadays techniques enable to combine ancient and modern methods. However, as these elements act as weak points of the structures during seismic events, the necessity of evaluating their vulnerability and safety level pushed the research to implement new numerical approaches. Although, the behavior of such structures is still not deeply investigated in literature as the high number of variables and uncertainty involved.

An innovative discrete homogenized model approach is here proposed. The method provides the main features needed for proper simulation of masonry curved structures, including the orthotropy and the typical in-and-out-of-plane coupled behavior exhibited by masonry vaults. Moreover, homogenization procedures directly implemented in the method allows reducing by far the number of variables, leading to non-linear analyses without unpractical computational time.

The model is conceived as an assembly of elastic units joint by non-linear interfaces. These latter are modeled as bricks elements and Concrete Damage Plasticity is used for modeling non-linear mechanical properties, coming from homogenization procedures. The discrete mesh is obtained automatically by means of an ad-hoc script.

With the aim to validate the proposed approach, some non-linear simulations are carried out on examples of an unreinforced dome, of which experimental and numerical data are available, showing the reliability of the method and the accuracy of reproducing the evolution of the damage with a limited computational burden.
\end{abstract}

\section{INTRODUCTION}

In the last years increasing efforts have been addressed for evaluating the safety and the 
effective behavior of masonry historical buildings. Such constructions spread all over the world as monuments, churches, bridges, aqueducts and even as residential buildings. Due to the fact that such building technology was developed by several countries in different periods of mankind, the level of complexity and variety from the geometrical and material point of view is huge. The most evident features are the composite nature of the material called masonry. Brick or stones are arranged in a different configuration with or without the use of mortar. Both of these components may have a different geological origin or chemical composition, making the application of usual engineer simplifications not straightforward. This complexity is, in a sense, amplified when masonry curved elements are studied. Arches, vaults and domes may be seen as the most identifying features of masonry buildings. However, their beauty is at least equal to their vulnerability, above all when subjected to horizontal seismic loads. In such events, catastrophic human and artistic lost increases the awareness of all technicians and pushed the researchers to carry out more experimental tests and implement innovative numerical approaches. It is important to highlight, as done by Huerta in [1] that, nowadays, vaults and domes are not any more built and the subsequent lack of knowledge have been filled only thank several researchers (see [2-4]) in the last decades. These latter identified, by means of a scientific approach, the effective behavior of curved masonry structures, laying the foundations to more advanced and complex methodologies. In fact, FE based approaches represent certainly the most valid option for properly exploring the performance until the collapse of such complex structures. Non-linear simulations might be carried out by means of micro and macro modeling. The first approach relies on separate
modeling of each component of masonry as stones, joints and interfaces (even of the
reinforcement when strengthening is simplated [5]). Such an approach dnsures detailed and
realistic reproduction of reality. Howeve, the main drawback is obvious y the high number of
variables involved and the consequent unpractical computational burden. A macro-modeling
approach may tackle these issues, leading to dasier implementation of the geonetrical model and reducing by far the numerical complexity. Some constitutive models were implemented in

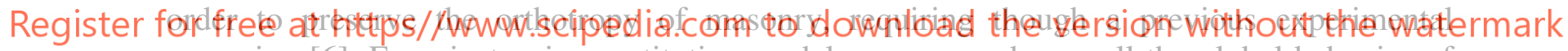
campaign [6]. Even isotropic constitutive models may reproduce well the global behavior of masonry structures [7], but at the same time, they can lead to possible inaccuracy in the evolution of the damage pattern.

Along with non-linear approaches, the kinematic limit analysis combined with FE or with NURBS [8-10] provides a speedy and straightforward approach for identifying the ultimate state at the collapse, allowing fast identification of the safety level. However, no information about the displacement and crack history can be obtained, precluding its application in some scenarios.

In order to address the drawbacks of the above-discussed methods, an innovative methodology for non-linear analysis of curved masonry structures is here proposed. It relies on a discrete model approach coupled with homogenization procedures. Moreover, the nonlinear mechanical properties are modeled by means of a generic damage-plasticity constitutive model already available in FE based software as Abaqus. Eventually, experimental and numerical comparisons are provided for assessing the validity of the method and for illustrating its capabilities. 


\section{FEATURES OF THE PROPOSED METHOD}

The method has been implemented with the aim to take into account properly all the aspects peculiar to masonry, preserving at the same time the computational effort, the accuracy and an easy implementation. First of all, a non-linear approach is selected in order to comply with the requirement of international codes that often ask for displacement history of the structure, such as pushover analysis. Then, above all for vaults and domes, due to the great heterogeneity of masonry textures, the orthotropy in the elastic and inelastic range should be reproduced. Moreover, when curved masonry structures are treated, the influence of the membrane state of stress on the out-of-plane behavior is a key aspect of a reliable and predictive method. Last but not least, the number of variables involved has to be kept low, with the way to be easily employed by practitioners. Orthotopy is well reproduced when a micro-modeling approach is selected. However, as mentioned, its use is limited to small scale samples.

A successful way to overcome such limitation is represented by homogenization procedures, often applied to composite materials like masonry. Such procedures are based on the identification of the global structure as a repetition of a representative volume element (RVE), enable to represent accurately the macroscopic behavior. In such a way the efforts required by micro approaches are limited only to a cell level, defining the elastic and inelastic response. Alternatively, a valid and speedy approach at the cell level is the one implemented and presented in [11]. A semi-analytical procedure is described, resulting highly effective and

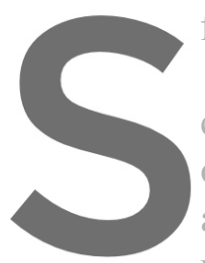
fast through the use of a

At the struetural can be input as an orthotropic mechanid alternative is offered units are linked by interfaces

commonly used method "RBSM" (Rigid Body Spring Approach), such interfaces are

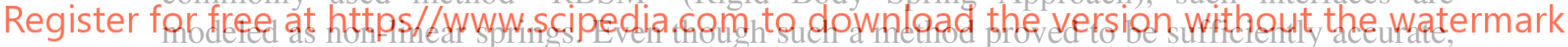

its application has been limited to walls loaded in-plane and out-of-plane, like the impossibility to keep coupled the in-and-out-of-piane effects. Moreover, the impiementation of the numerical model may result cumbersome.

The authors believe that a discrete approach along with homogenization procedures may lead to a predictive and straightforward method for modeling of masonry vaults and domes. For this reason, a new method based on the different implementation of the interfaces is here presented. The procedure follows the one described in [12] by the Authors. In this latter, the interfaces have been modeled as flat 3D bricks elements assigning the homogenized nonlinear material properties by means of the plastic-damage constitutive model CDP (concrete damage plasticity), already available in Abaqus. The structure is described as a repetition of elastic units, jointed by the interfaces. The choice to assign the elastic properties directly to the cells ensures improved stability when the inelastic range is reached.. Such 3D approach ensures that the influence of the normal stress related to the gravity loads on the non-linear behavior in flexion is automatically taken into account. The number of variables involved in the numerical simulations is very low when compared to micro approaches, opening the way to further application of the method on reinforced structures. 


\section{METHODOLOGY}

The validation of such discrete method on UR and RE out-of-plane-loaded walls is available in [12] where numerical and experimentally comparison is provided, and the interested readers are directed to this research for a point of reference.

The use of Concrete Damage Plasticity for modeling non-linear interfaces leads to easy implementation of the homogenized mechanical parameters along with robust numerical stability. CDP is as isotropic, plasticity-based, damage model, originally conceived for concrete. Successively it has been widely used for modeling masonry structures [13] in the static and dynamic non-linear analysis. A differential behavior in tension (exponential softening) and compression (parabolic softening) is assigned. The progressive decrease of the elastic stiffness is governed by two damage variables $\mathrm{dt}$ and $\mathrm{dc}$, which range from 0 (undamaged material) to 1 (completely damaged material).

It is important to highlight that through this approach, despite using a continuum constitutive model, the orthotropy results preserved as the values of non-linear parameters are defined according to the orientation of interfaces. Because of that, its application and a proper simulation of materials like-masonry is possible even in FEM software that does not have in their library orthotropic damage-based models.
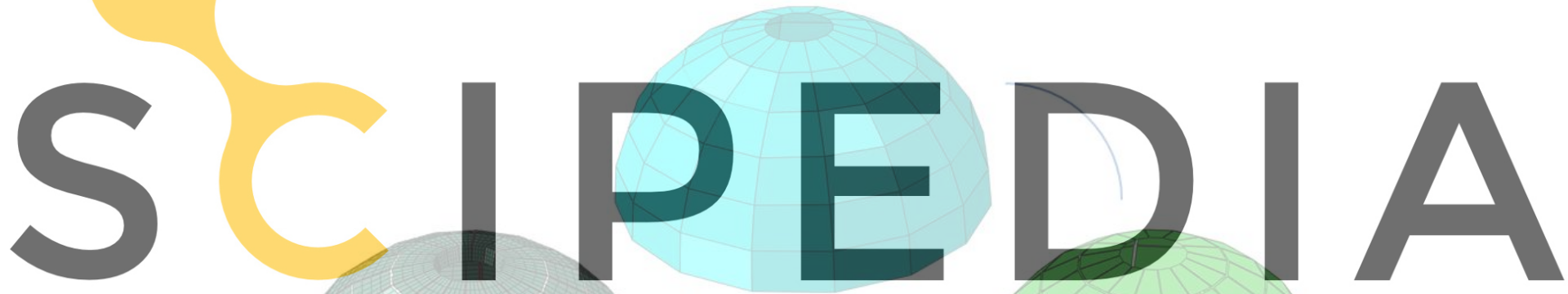

Initial inner-surface mesh

Register for free at https//www.scipedia.com to download the version without the watermark

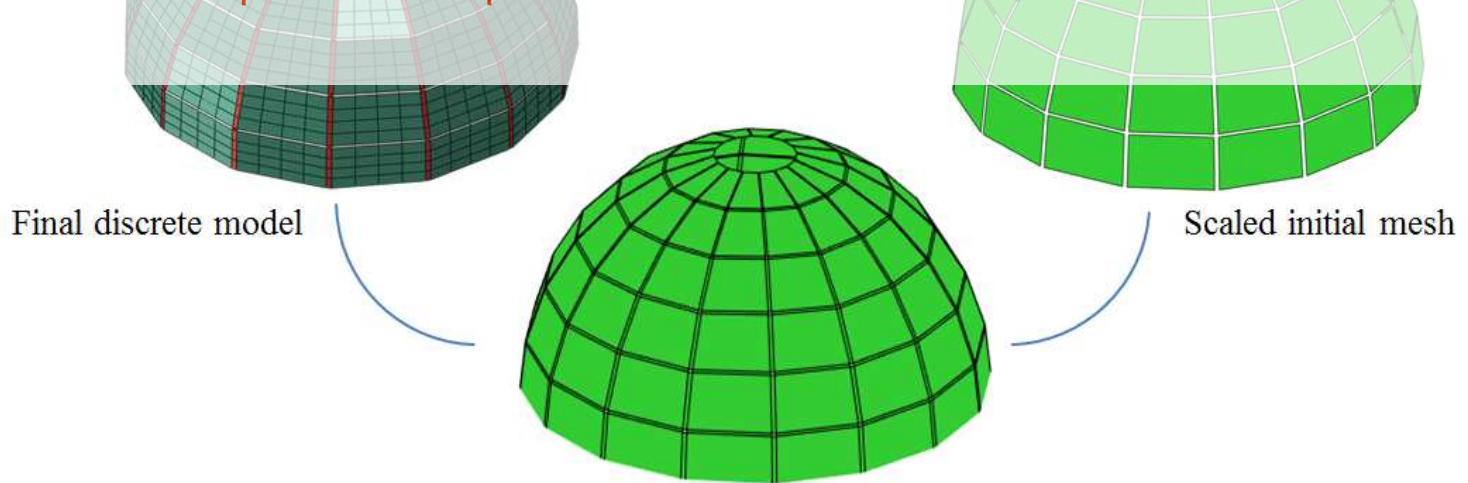

Automatic creation of interfaces

Figure 1: Steps for the automatic implementation of the discrete mesh.

The extension of this method to curved masonry structures is here presented and validated by experimental and numerical comparison of a brick-dome tested by Foraboschi in 2006. Moreover, the method has been improved by means of a MATLAB script that interacts directly with ABAQUS. The script, implemented by the Authors, is able to provide in an 
automatic way the discretized mesh and to assign automatically the mechanical homogenized properties to each interface. Such automatic procedure allows the overcoming of some difficulties during the preparation of the numerical method. In fact, the flat 8-noded brick elements employed as interfaces have a thickness that, even though negligible, implies the presence of gaps in the intersections.
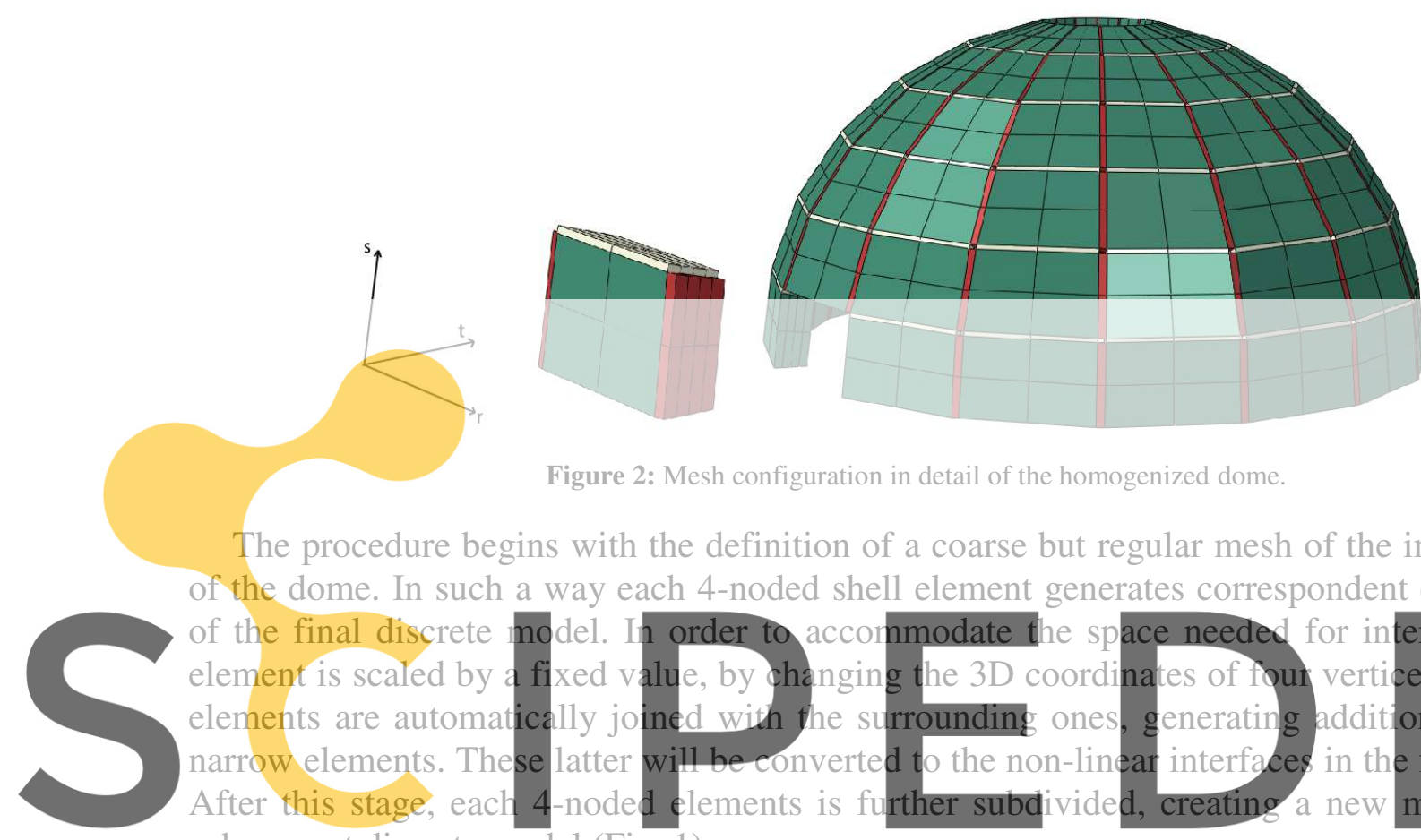

Figure 2: Mesh configuration in detail of the homogenized dome.

The procedure begins with the definition of a coarse but regular mesh of the inner surface of the dome. In such a way each 4-noded shell element generates correspondent elastic units of the final discrete model. In order to accommodate the space needed for interfaces, each element is scaled by a f elements are automatic narrow elements. These After this stage, each 4 subsequent discrete model (Fig. 1).
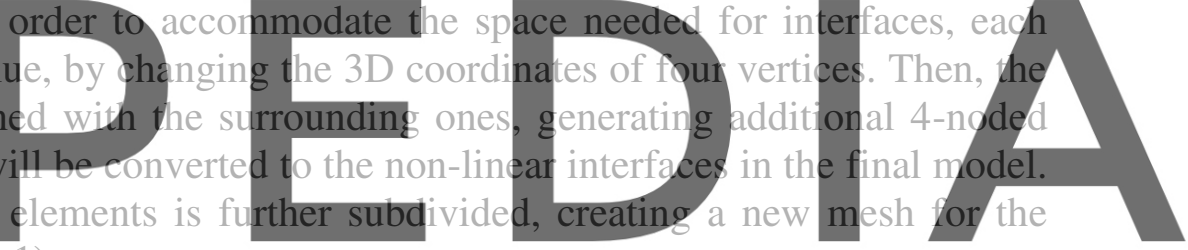

Register for The final madel is obtained by an extrusion gf the discrete shell mesh. A different quantity can be applied to each node, making possible the modeling of vaults with variable thickness.

In this paper, the dome has been modeled with 96 elastic units, providing two different discretizations. The first one with a rough mesh of 4 elements on the plane r-s. The second one, more refined, presents 16 elements on the plane r-s (Fig. 2).
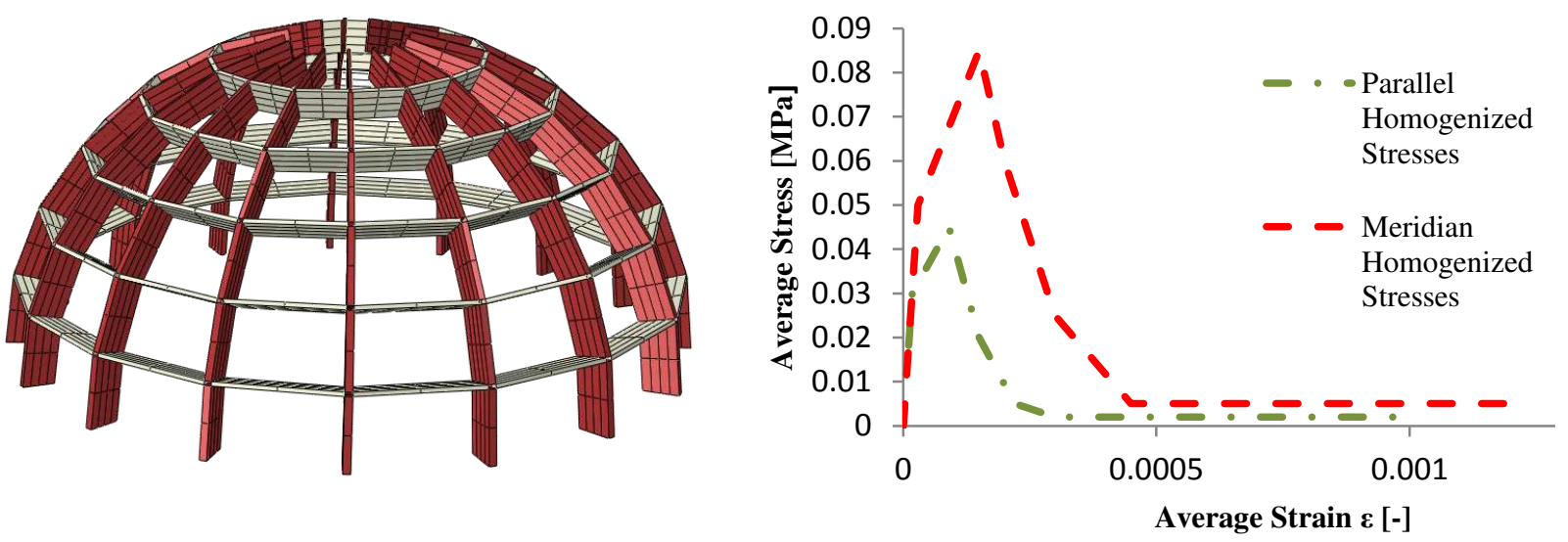

Figure 3: Detail of non-linear interfaces (Left) and relative homogenized stress-strain curve of tensile behavior (Right) 
For both models, 4 elements along the thickness of the dome are defined. This choice results sufficiently accurate, according to the experience of the Authors, for reproducing properly the flexural behavior of curved masonry structures. As mentioned, the homogenized mechanical properties are automatically assigned to the interfaces according to their orientation.

This possibility paves the way to a full and comprehensive homogenization procedure, taking advantage of a semi-analytical approaches that may be easily integrated within the script. Such an extension will be a future improvement of the method and the Authors refer to upcoming research the interested readers.

Here the numerical analysis (non-linear and limit analyses) carried out in [14] are taken as references. With the aim to be fully consistent, the homogenized parameters carried out at the cell level of the dome in [14] are re-applied and set by means of a stress-strain input in the Concrete Damage Plasticity (Fig. 3).

\section{RESULTS}

The dome used as a reference for the numerical analyses was tested by Foraboschi in 2006. It was made of typical Italian bricks with dimensions of $120 * 250 * 55(\mathrm{~mm})$, with an overall dimension of $2.2 \mathrm{~m}$ for the inner diameter and a thickness of $120 \mathrm{~mm}$. The dome was tested under the application of an increasing load along the border of the upper hole. The comparison is made in terms of load-displacement curves available from the test ad from

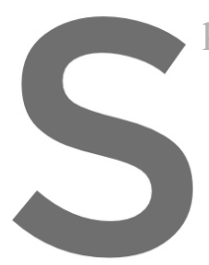
previous numerical analyses.
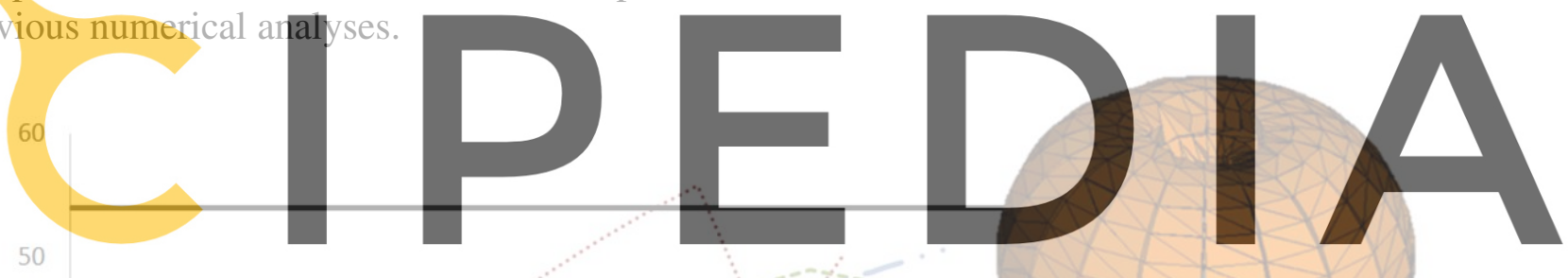

Register for free at https//www.scipedia.com to download the version without the watermark

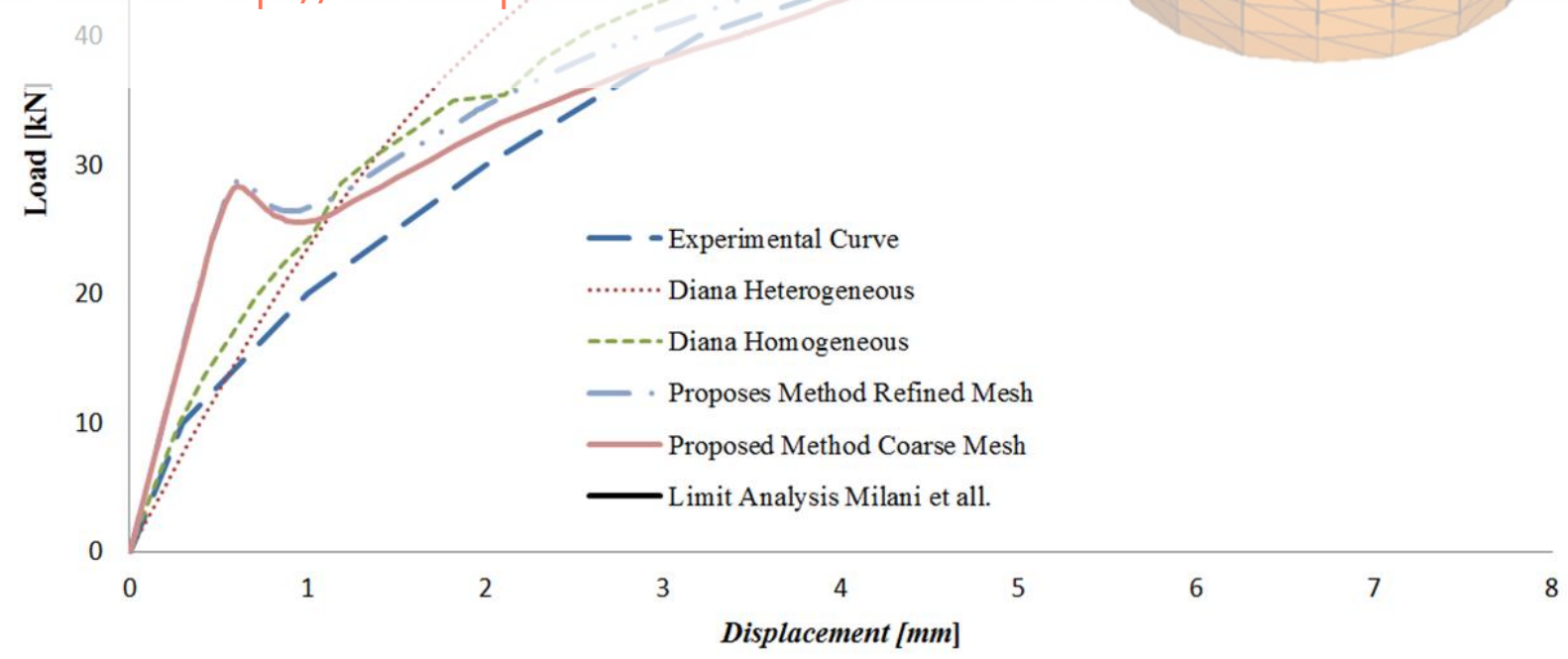

Figure 4: Comparison in terms of load-displacement curves of the proposed method with previous numerical analysis and with experimental reference; Collapse mechanism obtained by means of SQ non-linear analysis by

Milani, 2011 (Top right) 



Figure 5: Evolution of damage pattern

The global behavior presents a final displacement around $4.5 \mathrm{~mm}$ in correspondence of a peak capacity load nearby to $48 \mathrm{kN}$ (Fig. 4). Concerning the collapse mechanism, the final damage pattern is then compared with the one provided in [14] by the SQ non-linear analysis. As mentioned before, two different mesh configurations have been carried out for the purpose. The coarse one with a number of elements close to 3000 and the more refined one with almost 9000 elements. From Figure 4 it is noticeable how the homogenized outcome has an excellent capacity to reproduce the experimental and advanced numerical results, despite an initial discrepancy in the stiffness.

The damage and its evolution are coherent with what is expected from problem. In fact, the cracks star height. Along with meridian c the development of a circular

\section{CONCLUSION}


Numerical validation of a 3D discrete homogenized approach for the analysis of curved

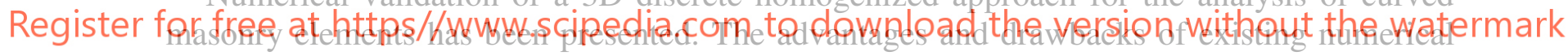
methods have been put in evidence with the aim to justify the implementation of a new method. The required features are so exposed: non-linear approach, low number of variables involved, orthotropic model, accuracy in the simulation of the influence of membrane stress on the flexural behavior. Consequently, a discrete approach coupled with homogenization procedures is suggested as the right methodology for fulfilling all the requirements.
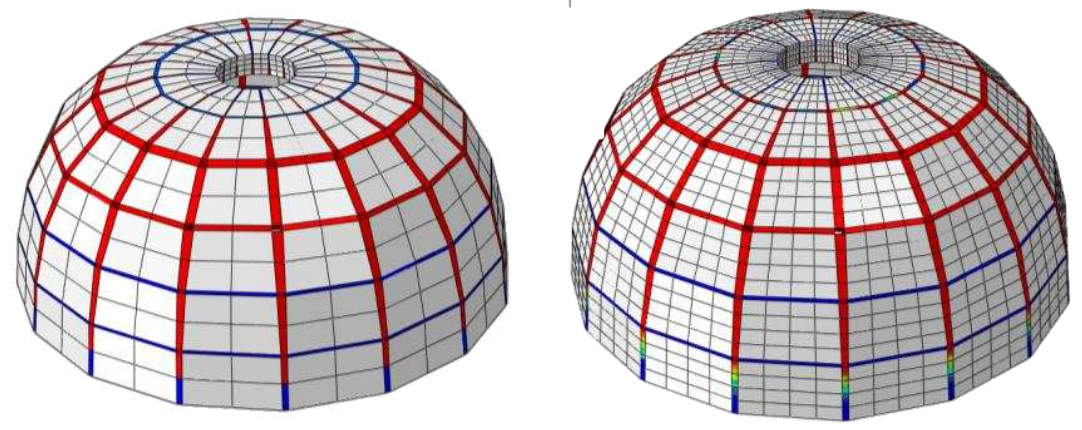

Figure 6: Comparison in terms of crack pattern between two different mesh configuration. 
The structure is modeled as repetition of elastic units jointed by non-linear interfaces. These latter are input as flat 8-noded bricks along with plastic, damage based, the constitutive model already available in Abaqus (concrete damage plasticity). In such a way, differential homogenized properties are assigned depending on the orientation of interfaces, preserving the orthotropic behavior. Then, a script for the realization of the geometric discrete model is presented, focusing on the possibility to obtain automatically the discretized mesh and opening the way to future extension.

Finally, the method, already validated on UR and RE out-of-plane-loaded walls [12], is extended to the case of a dome, experimentally tested in 2006 and numerically simulated several times by different approaches. The results obtained show good accuracy in replicating the global behavior in terms of the load-displacement curve, and in terms of damage pattern.

For further application, it is worth noting the negligible difference in the accuracy among the two different mesh configurations (Fig. 5-6). This result ensures the stability and validity of the method even when few variables are involved in the analysis, leading to speedy and reliable applications for practical purposes. Moreover, given the low computational burden, interesting extension to reinforced curved elements may be proposed by the Authors as topic of future works.

\section{REFERENCES}
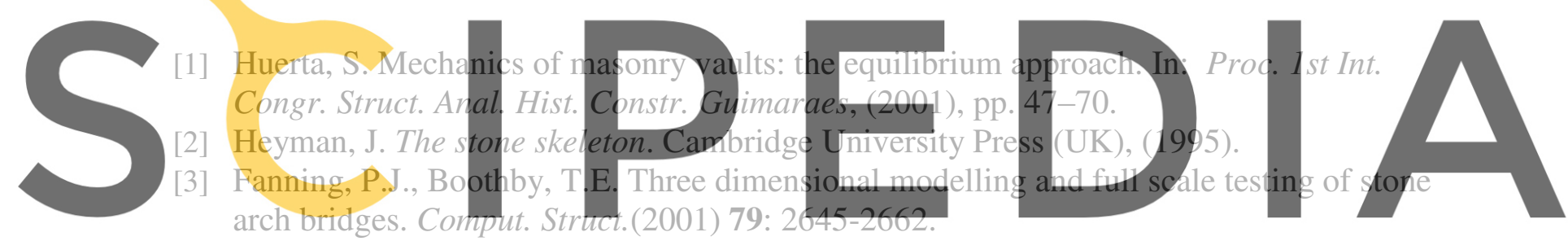

[4] Como, M. Statics of historic masonry costructions. Springer-Verlag Berlin (Germany),

Register for freelat, https//www.scipedia.com to download the version without the watermark

[5] Bove, M., Castellano, A., Fraddosio, A., Scacco,J., Milani, G. and Piccioni, M. D.

Experimental and Numerical Analysis of FRCM Strengthened Parabolic Tuff Barrel

Vault. In Key Engineering Materials, 817 (2019), pp. 213-220.

[6] Lourenço, P. B., De Borst, R. and Rots, J. G. A plane stress softening plasticity model for orthotropic materials. Int. J. Numer. Methods Eng. (1997) 40: 4033-4057.

[7] Bianchini, N., Mendes,N. and Lourenco, P.B. Seismic Assessment Of Masonry Cross Vaults Through Numerical Seismic Assessment Of Masonry Cross Vaults Through Numerical Nonlinear Static And Dynamic Analysis. In: COMPDYN 2019 7th International Conference on Computational Methods in Structural Dynamics and Earthquake Engineering, (2019).

[8] Grillanda, N., Chiozzi, A., Milani, G. and Tralli, A. Collapse behavior of masonry domes under seismic loads : An adaptive NURBS kinematic limit analysis approach. Eng.

Struct. (2019) 200.

[9] Chiozzi, A., Grillanda, N., Milani, G. and Tralli, A. UB-ALMANAC: An adaptive limit analysis NURBS-based program for the automatic assessment of partial failure mechanisms in masonry churches. Eng. Fail. Anal. (2018) 85: 201-220. 
[10] Grillanda, N., Chiozzi, A., Milani, G. and Tralli, A. On Collapse Behavior of Reinforced Masonry Domes under Seismic Loads. In Key Engineering Materials, 817 (2019), pp. 275-282.

[11] Bertolesi, E., Milani, G. and Lourenço, P. B. Implementation and validation of a total displacement non-linear homogenization approach for in-plane loaded masonry. Comput. Struct. (2016) 176: 13-33.

[12] Scacco, J., Ghiassi, B., Milani, G. and Lourenço, P.B. A fast modeling approach for numerical analysis of unreinforced and FRCM reinforced masonry walls under out-ofplane loading. Compos. Part B (2019) 180.

[13] Valente, M. and Milani, G. Effects of Geometrical Features on the Seismic Response of Historical Masonry Towers. J. Earthq. Eng. (2018) 22: 2-34.

[14] Milani, G. and Tralli, A. A simple meso-macro model based on SQP for the non-linear analysis of masonry double curvature structures. Int. J. Solids Struct. (2012) 49: vol. 808-834.
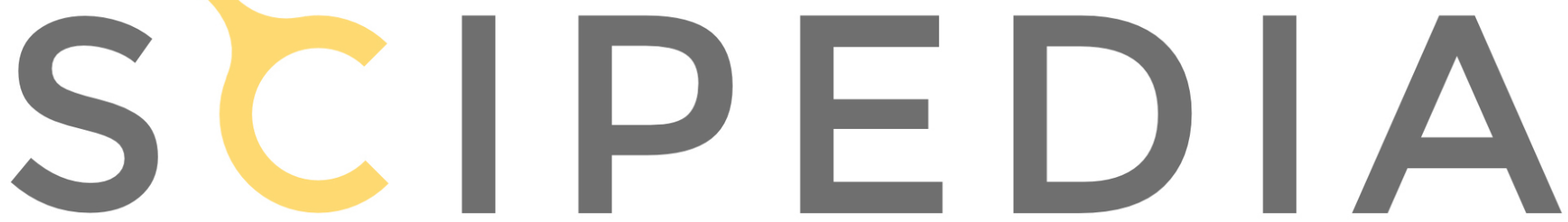

Register for free at https//www.scipedia.com to download the version without the watermark 